\title{
Los síntomas de sueño son predictores del número de comorbilidades. Estudio poblacional
}

\author{
Luis Torre-Bouscoulet, Rosario Fernández-Plata, David Martínez-Briseño, Rogelio Pérez-Padilla, \\ Abigail Guzmán-Barragán, Ma. Cecilia García-Sancho $\bowtie$
}

Instituto Nacional de Enfermedades Respiratorias Ismael Cosío Villegas, Ciudad de México.

Trabajo recibido: 06-I-2016; aceptado: 18-II-2016

\begin{abstract}
RESUMEN. Introducción: Los trastornos del dormir son un problema mundial de salud pública debido a que son causa frecuente de morbilidad y mortalidad. La asociación entre los síntomas relacionados al sueño y tipo y número de comorbilidades ha sido poco estudiada. Objetivo: Identificar si la presencia de síntomas relacionados al sueño predicen el tipo y número de comorbilidades en adultos $>40$ años, residentes del Área Metropolitana de la Ciudad de México. Métodos: Este informe deriva del proyecto PLATINO (Proyecto Latinoamericano para la Investigación de la Obstrucción Pulmonar) e incluye los datos obtenidos del muestreo poblacional de la Ciudad de México (número de aprobación C02-03). Los síntomas relacionados al sueño se obtuvieron de un cuestionario estandarizado (www.platino-alat.org/docs/ cuestionario_platino_mexico.pdf). Las comorbilidades registradas en este estudio fueron: enfermedades del corazón, hipertensión arterial sistémica, diabetes tipo 2, accidente vascular cerebral y gastritis y úlcera. Para identificar si los síntomas relacionados al sueño fueron predictores del número de comorbilidades utilizamos la regresión logística multinomial, considerando como variable dependiente a tres grupos: (0) personas sin síntomas relacionados al sueño; (1) sujetos con insomnio y uso regular de sedantes y (2) sujetos con síntomas de apnea obstructiva del sueño. El análisis se ajustó por el diseño del estudio y se realizó en el programa estadístico STATA versión 9.0. Resultados: La tasa de respuesta en el estudio fue $73.1 \%$. Las comorbilidades fueron muy frecuentes, $61.2 \%$ refirieron cuando menos una comorbilidad. El principal predictor de síntomas de sueño fue el número de comorbilidades que presentaba cada individuo tanto en el grupo de pacientes con insomnio y uso regular de sedantes: [RRR = 1.5 (IC95\% 1.3-1.7), p < 0.0001], como en el grupo de síntomas de AOS [RRR = 1.6 (IC95\% 1.1-3.3), $p=0.02$ ]. Conclusiones: Después de controlar por género, índice tabáquico, obesidad y la presencia de síntomas de ansiedad y depresión, los síntomas de sueño fueron el principal predictor independiente del número de comorbilidades. Aplicando un cuestionario sencillo, podríamos identificar a los individuos con múltiples comorbilidades y con un riesgo particularmente alto de síntomas relacionados al sueño que requieren tratamiento urgente y de esta manera, ser referidos a clínicas especializadas en sueño.
\end{abstract}

Palabras clave: Comorbilidades SAOS, sueño, adultos.

ABSTRACT. Introduction: Sleep disorders are a global public health problem because they are common cause of morbidity and mortality. The association between sleep-related symptoms, and type and number of comorbidities has been scarly studied. Objective: To identify whether the presence of sleep-related symptoms predict the type and number of comorbidities in adults $>40$ years, residents of the metropolitan area of Mexico City. Methods: This report derives from PLATINUM project (Latin American Project for the Investigation of Pulmonary Obstruction) and includes data from the sampling population of the city of Mexico (approval number C02-03). Sleep-related symptoms were collected from a standard questionnaire (www.platino-alat.org/docs/ cuestionario_ platino_mexico.pdf). Comorbidities recorded in this study were: heart disease, hypertension, type 2 diabetes, stroke and gastritis and ulcers. To identify whether the symptoms were sleep-related predictors of the number of comorbidities, we use multinomial logistic regression, considering three-group dependent variable (0) People without sleep-related symptoms; (1) subjects with insomnia and regular use of sedatives and (2) patients with symptoms of obstructive sleep apnea. The analysis was adjusted considering the design of the study and performed the statistical program STATA version 9.0. Results: The response rate in the study was $73.1 \%$. Comorbidities were very common, $61.2 \%$ reported at least one comorbidity; the main predictor of symptoms of sleep was the number of comorbidities found in each individual both in the group of patients with insomnia and regular use of sedatives [RRR $=1.5$ (95\% $\mathrm{Cl} 1.3-1.7), \mathrm{p}<0.0001]$, as in the group of symptoms of OSA [RRR $=1.6(95 \% \mathrm{Cl} 1.1-3.3), p=0.02]$. Conclusions: After controlling for gender, smoking history, obesity and the presence of symptoms of anxiety and depression, symptoms of sleep was the main independent predictor of the number of comorbidities. Applying a simple questionnaire, we could identify individuals with multiple comorbidities and with a particularly high risk of sleep-related symptoms that require emergency treatment and thus be referred to clinics specializing in sleep.

Key words: Comorbidities OSAS, sleep, adults. 


\section{INTRODUCCIÓN}

Los trastornos del sueño son un problema global de salud pública debido a que son causa de morbilidad y mortalidad. ${ }^{1}$ Aunque existen más de cien trastornos del sueño reconocidos, el insomnio y la apnea obstructiva del sueño (AOS) son dos de los más prevalentes, y la evidencia epidemiológica ha demostrado sólidamente que existe asociación significativa entre dichos trastornos con enfermedades crónicas ${ }^{2-4} y$ muerte prematura. ${ }^{5}$

La edad y la obesidad, entre otros, son factores de riesgo que comparten algunos trastornos del sueño con las enfermedades cardiovasculares y metabólicas. ${ }^{6,7}$ Por lo tanto, podríamos anticipar que existe una elevada frecuencia de enfermedades comórbidas en pacientes con trastornos del sueño, lo que podría aumentar la carga para los sistemas de salud y conferir mayor riesgo de mortalidad. ${ }^{8,9}$

Previamente nuestro grupo publicó la prevalencia de síntomas relacionados al sueño en un estudio de base poblacional llevado a cabo en cinco grandes ciudades de América Latina. ${ }^{10}$ Tomando en cuenta esa información, nos planteamos el presente estudio con el objetivo de medir en adultos residentes de la Ciudad de México mayores de 40 años, la prevalencia de la coexistencia de síntomas asociados al sueño con enfermedades crónicas y analizar su potencial asociación. Si se probara esa asociación podríamos identificar-usando un cuestionario simple de síntomas relacionados a sueño- a los individuos con un riesgo particularmente alto de complicaciones que requieren tratamiento urgente $y$, de esta manera, ser referidos a clínicas especializadas en sueño.

\section{MÉTODOS}

Este informe es parte del estudio PLATINO (Proyecto Latinoamericano para Investigar la Obstrucción Pulmonar). PLATINO es una encuesta transversal, con muestreo poblacional en múltiples etapas que incluyó información de la Ciudad de México (México), Montevideo (Uruguay), Santiago (Chile), San Paulo (Brasil) y Caracas (Venezuela). Aquí, nosotros presentamos el análisis de la población residente en la zona metropolitana de la Ciudad de México. La metodología de este estudio ha sido descrita previamente. ${ }^{11}$ Brevemente, el muestreo fue de tipo probabilístico llevado a cabo en varias etapas manteniendo la representatividad poblacional. Mediante la ecuación de la precisión de una proporción estimamos el número de sujetos que debían ser incluidos. El cálculo del tamaño de muestra sugirió que 1,000 personas deberían seleccionarse en cada área con un margen de error entre 2 y $4 \%$. El enfoque fue localizar a 1,200 personas por sitio, con una tasa de no respuesta predicha de $20 \% .{ }^{4}$ El estudio fue aprobado por el Comité Institucional de Ciencia y Bioética del Instituto Nacional de Enfermedades Respiratorias Ismael Cosío Villegas (número de aprobación C02-03).

El cuestionario PLATINO (www.platino-alat.org/docs/ cuestionario_platino_mexico.pdf) es un instrumento estandarizado que explora factores de riesgo para enfermedades respiratorias, síntomas respiratorios, síntomas relacionados al sueño y enfermedades crónicas. La encuesta se aplicó a los sujetos seleccionados por personal previamente entrenado y estandarizado. Además de la encuesta, se realizó una espirometría forzada (Easy-One; ndd Medizintechnik AG, Zurich, Switzerland) de acuerdo con los estándares internacionales $2005,{ }^{12}$ y se obtuvieron mediciones antropométricas por duplicado mediante un estadímetro (208 stadiometer Seca Corporation, USA) y una cinta métrica inextensible (fiberglass tape measure); el peso fue obtenido por duplicado mediante una báscula electrónica (solar scale HS-301 Tanita Corporation, Inc., USA). El índice de masa corporal (IMC) se calculó mediante la fórmula peso/talla ${ }^{2}$ y se categorizó en normal (IMC $\leq$ 24.9); sobrepeso ( $\geq 25$ y $\leq 29.9$ ); obesidad $(\geq 30$ y $\leq$ 39.9) y obesidad mórbida $(\geq 40)$.

En el cuestionario los síntomas asociados al sueño que se exploraron fueron: apneas observadas, ronquido habitual (todas o casi todas las noches), somnolencia excesiva diurna (SED), insomnio (dificultad para iniciar o mantener el sueño todas o casi todas las noches) y uso de hipnóticos. La presencia de apneas observadas, ronquido habitual y SED fueron considerados como síntomas relacionados a AOS. Los síntomas de ansiedad y depresión fueron tomados del cuestionario breve de calidad de vida (SF-12) que también se encuentra integrado al cuestionario PLATINO. Los síntomas fueron analizados como variables categóricas (sí o no).

Las enfermedades comórbidas fueron las autorreferidas por el entrevistado (diagnóstico previo hecho por un médico). La pregunta del cuestionario PLATINO fue, ¿alguna vez en la vida el médico le ha dicho que usted tenía alguna de las siguientes enfermedades?: enfermedades del corazón, hipertensión arterial sistémica, diabetes mellitus, enfermedad vascular cerebral y gastritis o úlcera. Se registró el número de enfermedades comórbidas de cada sujeto de 0 a 5.

\section{Análisis estadístico}

Las variables numéricas se muestran en medias y desviación estándar, mientras que las dicotómicas en frecuencias y porcentajes. La comparación entre grupos se realizó mediante la prueba to $\chi^{2}$. Para el análisis 
multivariado utilizamos regresión logística multinomial. Las variables dependientes fueron tres grupos: (0) personas sin síntomas relacionados al sueño; (1) sujetos con insomnio y uso regular de hipnóticos y (2) sujetos con síntomas de AOS (ronquido habitual, somnolencia excesiva diurna y apneas observadas). En el análisis el grupo sin síntomas relacionados al sueño fue el grupo de referencia. Se consideró como significativo un valor $p<0.05$ bimarginal. En el modelo multinomial se reportaron las asociaciones como razones de riesgo relativos (RRR). El análisis estadístico se realizó con el programa estadístico STATA versión 9.0.13

\section{RESULTADOS}

El total de sujetos incluidos fue de 1,063 con una tasa de respuesta de $73.1 \%$. La edad promedio fue de 55.9 \pm 11.9 años y $613(57.6 \%)$ fueron mujeres. Identificamos a 650 sujetos (61.2\%) que refirieron al menos alguna enfermedad incluida en el cuestionario: enfermedades del corazón, hipertensión arterial sistémica, diabetes mellitus, enfermedad vascular cerebral y gastritis o úlcera.

La tabla 1 muestra las características generales de los sujetos incluidos, así como la prevalencia de enfermedades crónicas de acuerdo con los síntomas relacionados al sueño. El número de sujetos que no reportaron síntomas relacionados al sueño fue de 663 (62.4\%); los que refirieron insomnio y uso regular de sedantes 369 (34.7\%) y los que presentaron síntomas de AOS fueron 31 (2.9\%). Estos grupos fueron mutuamente excluyentes y en el análisis se consideró como grupo de referencia a los sujetos que no refirieron síntomas relacionados al sueño. Las condiciones que resultaron significativamente diferentes entre los grupos fueron el sexo masculino, tabaquismo (paquetes/año), IMC y obesidad, hipertensión arterial sistémica, enfermedades del corazón y síntomas de ansiedad y depresión.

En nuestra población, sin tomar en cuenta los síntomas de sueño, 61.2\% (650/1062) aceptó tener al menos una enfermedad comórbida; sólo 38.8\% (412/1063) refirieron no ser portadores de alguna enfermedad asociada. Del grupo completo, 402/1063 (37.8\%) tuvieron una sola comorbilidad; $181 / 1063$ (17.0\%) dos comorbilidades; 53/1063 (5.0\%) tres comorbilidades; y $14 / 1,063(1.3 \%)$ cuatro o más comorbilidades (tabla 2).

En el total de la población, las enfermedades crónicas que tuvieron mayor prevalencia fueron: hipertensión arterial sistémica (31.2\%); gastritis y úlcera (33.6\%) diabetes mellitus tipo 2 (15.2\%); enfermedades del corazón (10.5\%); la prevalencia de enfermedad vascular cerebral fue de $1.7 \%$. La media y desviación estándar del IMC de toda la población de estudio fue de $28.8 \pm 5.0 \mathrm{~kg} / \mathrm{m}^{2}$.

La tabla 2 muestra el promedio y número de comorbilidades con relación a la presencia de síntomas relacionados al sueño. El promedio de comorbilidades fue significativamente mayor entre los grupos que refirieron uso de sedantes e insomnio y el grupo con síntomas de AOS en comparación con el grupo sin síntomas ( $p$ $<0.0001$ ).

La tabla 3 muestra los resultados del análisis multivariado mediante regresión logística multinomial. El

Tabla 1. Características generales y prevalencia de enfermedades crónicas de acuerdo con los síntomas relacionados al sueño. Los datos son número y porcentaje a menos que se especifique otra cosa.

\begin{tabular}{|c|c|c|c|c|}
\hline Característica & $\begin{array}{l}\text { Sin síntomas relacionados } \\
\text { al sueño }(n=663)\end{array}$ & $\begin{array}{l}\text { Insomnio-sedantes } \\
\qquad(n=369)\end{array}$ & $\begin{array}{l}\text { Síntomas de AOS } \\
\qquad(n=31)\end{array}$ & Valor de $p$ \\
\hline Edad, años* & $56.1 \pm 12.1$ & $55.4 \pm 11.6$ & $58.4 \pm 12.8$ & 0.3 \\
\hline Hombres & $318(48)$ & 113 (30.6) & 19 (61.3) & $<0.0001$ \\
\hline Tabaquismo, paquetes/año* & $2.1 \pm 8.0$ & $2.1 \pm 7.9$ & $10.2 \pm 17.8$ & $<0.0001$ \\
\hline $\mathrm{IMC}, \mathrm{k} / \mathrm{m}^{2 *}$ & $28.6 \pm 5.1$ & $28.9 \pm 4.9$ & $30.7 \pm 6.3$ & 0.04 \\
\hline Obesidad & $216(32.6)$ & 141 (38.2) & $16(51.6)$ & 0.02 \\
\hline Obesidad mórbida & $25(3.8)$ & $13(3.5)$ & $3(9.7)$ & 0.2 \\
\hline Enfermedades del corazón & $56(8.5)$ & $47(12.7)$ & $9(29.0)$ & $<0.0001$ \\
\hline Hipertensión arterial sistémica & $178(26.9)$ & 139 (37.7) & $15(48.4)$ & $<0.0001$ \\
\hline Diabetes mellitus tipo 2 & $96(14.5)$ & $63(17.1)$ & $3(9.7)$ & 0.4 \\
\hline Enfermedad cerebral vascular & $11(1.7)$ & $6(1.6)$ & $1(3.2)$ & 0.8 \\
\hline Gastritis y úlcera & $170(25.6 \%)$ & $173(46.9)$ & $14(45.2)$ & $<0.0001$ \\
\hline Ansiedad/depresión & $6(0.9)$ & $23(6.2)$ & $2(6.5)$ & $<0.0001$ \\
\hline
\end{tabular}

* Media y DE.

AOS = Apnea obstructiva del sueño; IMC = Índice de masa corporal; obesidad: IMC $\geq 30 \mathrm{k} / \mathrm{m}^{2} ;$ obesidad mórbida: IMC $\geq 40 \mathrm{k} / \mathrm{m}^{2} ; \mathrm{HAS}=\mathrm{Hiper}-$ tensión arterial sistémica; DM2 = Diabetes tipo 2; EPOC = Enfermedad pulmonar obstructiva crónica. Los síntomas de ansiedad y depresión fueron tomados del cuestionario breve de calidad de vida (SF-12) que también se encuentra integrado al cuestionario PLATINO. 
uso regular de sedantes y el insomnio se asociaron al número de comorbilidades de los sujetos aun después de controlar por género, índice tabáquico, ansiedad, depresión y falta de energía y obesidad [RRR $=1.5$ (IC95\% 1.3-1.7), $p<0.0001]$. De manera similar, los síntomas de AOS se asociaron al número de comorbilidades del sujeto aun después de controlar por género, índice tabáquico, ansiedad, depresión y falta de energía y obesidad [RRR $=1.6$ (IC95\% 1.1-3.3), $p=0.02$ ]. Cada grupo de síntomas de sueño (insomnio y uso regular de sedantes, y síntomas de AOS), incrementó el riesgo de tener un mayor número de comorbilidades entre 50 y $60 \%$ (tabla 3 ).

\section{DISCUSIÓN}

Los principales resultados del estudio fueron: (1) las comorbilidades son muy frecuentes: $61.2 \%$ de los individuos refirieron cuando menos una; (2) en el total de la población, las enfermedades crónicas que tuvieron mayor prevalencia estuvieron: hipertensión arterial sistémica (31.2\%); gastritis y úlcera (33.6\%) diabetes mellitus tipo 2 (15.2\%); enfermedades del corazón (10.5\%); la prevalencia de enfermedad vascular cerebral fue de 1.7\%; (3) la prevalencia del número de comorbilidades fue diferente de acuerdo con el tipo de síntomas de sueño referidos; (4) la principal asociación entre la presencia de síntomas de sueño fue el número de comorbilidades que presentaba cada individuo, tanto en el grupo de pacientes con insomnio y uso regular de sedantes: [RRR = 1.5 (IC95\% 1.3-1.7), p < 0.0001] como en el de síntomas de AOS [RRR = $1.6($ IC95\% 1.1-3.3), $p=0.02]$.

La población residente de la Ciudad de México mostró una elevada prevalencia de enfermedades crónicas diagnosticadas por un médico: $61.2 \%$ de la población reportó al menos una enfermedad; $5 \%$ refirió tres comorbilidades y $1.3 \%$ reportaron cuatro o cinco comorbilidades. Las enfermedades crónicas que presentaron una prevalencia diferencial de acuerdo con el grupo de síntomas del sueño fueron: obesidad, hipertensión arterial sistémica, enfermedades cardiovasculares, gastritis y úlcera y ansiedad/depresión, así como factores de riesgo como el tabaquismo. El tipo

Tabla 2. Número de comorbilidades de acuerdo con los síntomas relacionados al sueño. Los datos son número y porcentaje a menos que se especifique otra cosa.

\begin{tabular}{|c|c|c|c|c|}
\hline Característica & $\begin{array}{l}\text { Sin síntomas relacionados } \\
\text { al sueño } \\
(\mathrm{n}=663)\end{array}$ & $\begin{array}{l}\text { Insomnio-sedantes } \\
\qquad(\mathrm{n}=369)\end{array}$ & $\begin{array}{l}\text { Síntomas de AOS } \\
\qquad(n=31)\end{array}$ & Valor de $p$ \\
\hline Número de comorbilidades* & $0.8 \pm 0.9$ & $1.2 \pm 0.9$ & $1.4 \pm 1.1$ & $<0.0001$ \\
\hline Sin comorbilidades & 307 (46.3) & $98(26.6)$ & 7 (22.6) & $<0.0001$ \\
\hline Una comorbilidad & 242 (36.5) & $148(40.2)$ & $12(38.7)$ & \\
\hline Dos comorbilidades & $80 \quad(12.1)$ & 94 (25.5) & 7 (22.6) & \\
\hline Tres comorbilidades & $27(4.1)$ & $22(6.0)$ & $4 \quad(12.9)$ & \\
\hline Cuatro o más comorbilidades & $7(1.1)$ & $6(1.6)$ & 1 (3.2) & \\
\hline
\end{tabular}

Síntomas de AOS (ronquido, somnolencia y apnea).

${ }^{*}$ Media \pm DE.

Tabla 3. Resultados del análisis por regresión logística multinomial. Correlatos asociados a síntomas de sueño.

\begin{tabular}{|c|c|c|c|c|c|c|}
\hline \multirow[b]{2}{*}{ Característica } & \multicolumn{3}{|c|}{$\begin{array}{l}\text { Insomnio y uso regular de sedantes } \\
\qquad(\mathrm{n}=369)\end{array}$} & \multicolumn{3}{|c|}{ 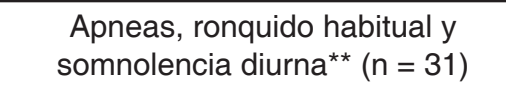 } \\
\hline & RRR & IC95\% & $\mathrm{p}$ & RRR & IC95\% & $p$ \\
\hline Hombre & 0.5 & $0.3-0.6$ & $<0.001$ & 1.6 & $0.7-3.8$ & 0.2 \\
\hline Paquetes año de tabaquismo & 1.003 & $0.9-1.02$ & 0.7 & 1.03 & $1.01-1.06$ & 0.002 \\
\hline Obesidad $^{*}$ & 1.03 & $0.8-1.4$ & 0.8 & 2.4 & $0.9-36.7$ & 0.05 \\
\hline Ansiedad/depresión (SF-12) & 5.1 & $2.0-13.0$ & 0.001 & 5.8 & $0.9-26.7$ & 0.06 \\
\hline Número de comorbilidades & 1.5 & $1.3-1.7$ & $<0.0001$ & 1.6 & $1.1-3.3$ & 0.02 \\
\hline
\end{tabular}

En el análisis multivariado se controló por el diseño del estudio. El grupo de referencia fue el grupo que no reportó síntomas de sueño $(\mathrm{n}=663)$. Las asociaciones las expresamos en la RRR.

$\mathrm{RRR}=$ Razón de riesgo relativo; IC95\% = Intervalo de confianza al 95\%.

* Índice de masa corporal $\geq 30 \mathrm{k} / \mathrm{m}^{2}$. 
y número de comorbilidades reportadas por esta población es alarmante ya que se trata de una población general, representativa de la Ciudad de México y área metropolitana, y con una media de edad relativamente baja, de 55.9 ( \pm 11.9 años). Los estudios que asocian la obesidad con las enfermedades crónicas son abundantes. ${ }^{14,15}$ Algunos autores sugieren que esta carga de enfermedad observada para los trastornos del dormir es también una carga debido a la obesidad.

En nuestro estudio el $20.5 \%$ de la población tuvo un IMC normal, $44.0 \%$ mostró sobrepeso, 31.6\% mostró obesidad y $3.9 \%$ obesidad mórbida. La suma de sobrepeso y obesidad en nuestra población representa el $75.6 \%$ de la población estudiada, cifra que semeja lo informado en la Encuesta Nacional de Salud y Nutrición del año 2012, en la cual entre población con edad igual o mayor a 20 años, la cifra en hombres fue de $69.4 \%$ y en mujeres de $73.0 \% .{ }^{16}$ En nuestra población, encuestada en el 2003, la prevalencia de obesidad y sobrepeso fue para las mujeres de $78.9 \%$ y para los hombres de $79.1 \%$. La prevalencia de síntomas de AOS en los grupos de sobrepeso, obesidad y obesidad mórbida fue de $87.1 \%$. La asociación entre obesidad y trastornos del sueño es bidireccional de acuerdo con estudios previos; la obesidad genera apnea del sueño y ésta, a su vez, aumenta la resistencia a la insulina. ${ }^{17} \mathrm{La}$ obesidad podría ser el factor que subyace la asociación entre diversas enfermedades comórbidas. ${ }^{18}$

El tabaquismo parece estar relacionado a la presencia de síntomas de AOS; sin embargo, otros estudios han fallado en demostrar esta asociación. ${ }^{19} \mathrm{Al}$ igual que lo demostrado por otros autores, el género masculino fue protector para insomnio y factor de riesgo para síntomas de AOS.

\section{Limitaciones del estudio}

Este estudio tiene varias limitaciones. La principal es inherente al diseño transversal del estudio, lo que nos impide afirmar la existencia de una relación causal entre el número de comorbilidades y los síntomas relacionados al sueño o viceversa. No obstante, nuestros hallazgos nos permiten generar hipótesis para estudios futuros. Otra limitación es que el cálculo de la prevalencia de síntomas relacionados al sueño se hizo con base en los síntomas autorreferidos a través de un cuestionario, por lo que no podemos afirmar que aún la coexistencia de somnolencia, apneas observadas y el ronquido habitual signifique que un paciente tiene SAOS. Aunque consideramos que los pacientes que perciben los síntomas asociados al sueño necesitan de una evaluación posterior para excluir dicho trastorno. De la misma manera, las comorbilidades son autorreferidas y no hicimos estudios adicionales para confirmarlas. El modelo logístico presentado en este estudio fue elaborado con un propósito exploratorio, por lo que es posible que otros factores de riesgo no bien conocidos pudieran estar involucrados en estas asociaciones.

No obstante, las principales fortalezas de nuestro estudio es que éste presenta información basada en una muestra poblacional tomada del Área Metropolitana y de la Ciudad de México y que obtuvo, además, una alta tasa de respuesta de $73.1 \% .^{1}$ Además, aun cuando nuestro estudio es de corte transversal usamos como medida de asociación la RRR. Esta medida permite calcular la magnitud de la asociación de una manera más precisa a la estimación de la razón de momios, lo cual hace más consistente la asociación observada en este análisis entre la presencia de síntomas de sueño y el número de comorbilidades.

\section{CONCLUSIONES}

Nosotros encontramos que la presencia de síntomas de sueño autorreferidos se asocia a un mayor número de comorbilidades también autorreferidas. Con base en los resultados de este estudio podríamos identificar -usando un cuestionario simple de síntomas relacionados a sueño- a los individuos con un riesgo particularmente alto de complicaciones que requieren tratamiento urgente y, de esta manera, ser referidos a clínicas especializadas en sueño.

\section{Declaración de intereses}

Este estudio fue propuesto por la ALAT (Asociación Latinoamericana del Tórax) y recibió un fondo para investigación de Boehringer-Ingelheim. Los autores declaran no tener conflicto de intereses. Este estudio también fue apoyado por el Consejo Nacional de Ciencia y Tecnología de México (CONACYT SALUD2004-CO1-72).

\section{REFERENCIAS}

1. Lavie L. Oxidative stress--a unifying paradigm in obstructive sleep apnea and comorbidities. Prog Cardiovasc Dis 2009;51:303-312.

2. Pamidi S, Aronsohn RS, Tasali E. Obstructive sleep apnea: role in the risk and severity of diabetes. Best Pract Res Clin Endocrinol Metab 2010;24:703-715.

3. Selim B, Won C, Yaggi HK. Cardiovascular consequences of sleep apnea. Clin Chest Med 2010;31:203-220.

4. Ryan S, Taylor CT, McNicholas WT. Systemic inflammation: a key factor in the pathogenesis of cardiovascular complications in obstructive sleep apnoea syndrome? Postgrad Med J 2009;85:693-698. 
5. Young T, Palta M, Dempsey J, Peppard PE, Nieto FJ, Hla KM. Burden of sleep apnea: rationale, design, and major findings of the Wisconsin Sleep Cohort study. WMJ 2009;108:246-249.

6. Ryan S, Crinion SJ, McNicholas WT. Obesity and sleepdisordered breathing--when two "bad guys" meet. QJM 2014;107:949-954.

7. Manna P, Jain SK. Obesity, oxidative stress, adipose tissue dysfunction, and the associated health risks: causes and therapeutic strategies. Metab Syndr Relat Disord 2015;13:423-444.

8. Tarasiuk A, Greenberg-Dotan S, Brin YS, Simon T, Tal A, Reuveni H. Determinants affecting health-care utilization in obstructive sleep apnea syndrome patients. Chest 2005;128:1310-1314.

9. Hopps E, Caimi G. Obstructive sleep apnea syndrome: links between pathophysiology and cardiovascular complications. Clin Invest Med 2015;38:E362-E370.

10. Bouscoulet LT, Vazquez Garcia JC, Muiño A, et al. Prevalence of sleep related symptoms in four Latin American cities. J Clin Sleep Med 2008;4:579-585.

11. Menezes AM, Victora CG, Perez-Padilla R; PLATINO Team. The Platino project: methodology of a multicenter prevalence survey of chronic obstructive pulmonary disease in major Latin American cities. BMC Med Res Methodol 2004;4:15.

12. Miller MR, Hankinson J, Brusasco V, et al. Standardization of spirometry. Eur Respir J 2005; 26:319-38.

13. Stata Technical Support. Citing Stata software, documentation, and FAQs; Date November 1999; updated June 2013.

14. Smith R, Ronald J, Delaive K, Walld R, Manfreda J, Kryger $\mathrm{MH}$. What are obstructive sleep apnea patients being treated for prior to this diagnosis? Chest 2002;121:164172.

15. Bonnefoy M, Gilbert T. Body composition and comorbidity in the elderly. Geriatr Psychol Neuropsychiatr Vieil 2015;13 Suppl 1:29-36.

16. Gutiérrez JP, Rivera-Dommarco J, Shamah-Levy T, Villalpando-Hernández S, Franco A, Cuevas-Nasu L, et al. Encuesta Nacional de Salud y Nutrición 2012. Resultados Nacionales. Cuernavaca, México: Instituto Nacional de Salud Pública (MX), 2012.

17. Badran M, Yassin BA, Fox N, Laher I, Ayas N. Epidemiology of sleep disturbances and cardiovascular consequences. Can J Cardiol 2015;31:873-879.

18. Piché MÈ, Auclair A, Harvey J, Marceau S, Poirier P. How to choose and use bariatric surgery in 2015. Can J Cardiol 2015;31:153-166.

19. Krishnan V, Dixon-Williams S, Thornton JD. Where there is smoke...there is sleep apnea: exploring the relationship between smoking and sleep apnea. Chest 2014;146:1673-1680.

\section{$\triangle$ Correspondencia:}

Dra. en C. Cecilia García-Sancho Instituto Nacional de Enfermedades Respiratorias Ismael Cosío Villegas. Calzada de Tlalpan Núm. 4502, colonia Sección XVI, 14080, Ciudad de México. Teléfono: 54-87-17-00, extensión 5238,

fax: $56-65-46-23$

Correo electrónico:

cegarsan@netscape.net; cegarsanfi@gmail.com

Los autores declaran no tener conflicto de intereses. 